of the cortical centres by the method of excitation. Hence the limits of a region may be somewhat differently given by different experimenters; but, making due allowance for all these sources of error, it has been found possible to arrive at a remarkable degree of harmony as to the locality and extent of the respective areas. The extra-polar diffusion of current which can be demonstrated in the brain, as in other animal tissues, has been regarded by Dupuy ${ }^{33}$ as an insuperable objection to the theory that the results of application of the electrodes to the cortex are due to stimulation of the cortex itself; and attempts are made to explain them away by mere physical conduction of the currents to centres and tracts at the base of the brain. But no satisfactory explanation can thus be afforded of the manifest differences in reaction which follow the application of the electrodes to regions in close proximity to each other, nor of the total absence of reaction when the electrodes are placed on the island of Reil, which is nearer the base of the brain than other regions which act uniformly and without fail.

The chief objection to the direct excitability of the cortex itself is found in the fact that, even after removal of the cortex, similar reactions are still obtainable when the electrodes are placed on the subjacent medullary fibres. This was first pointed out by Burdon-Sanderson, 31 and has been confirmed by all subsequent experimenters. After removal of the cortex, however, the medullary fibres lose their excitability, like motor nerves separated from the anterior cornua of the spinal cord, so that, after a lapse of four days, no reactions can be produced by the strongest stimulation. This fact completely disposes of the physicalconduction-to-the-base-of-the-brain hypothesis. It has thus been satisfactorily established that the cones of medullary fibres, corresponding to the respective cortical centres, are functionally differentiated like the cortical centres them selves, and, as has been shown by Franck and Pitres, ${ }^{35}$ and more recently, with greater detail, by Beevor and Horsley, ${ }^{36}$ maintain their individuality, and are écheloned in definite and regular order in the internal capsule.

On the accompanying figure (Fig. 4), kindly supplied me by Dr. Beevor, are marked the points on the internal capsule from which, according to their recent researches, minimal stimulation excites the respective movements which are indicated on the margin. But it does not follow because the medullary fibres are excitable that the corresponding cortical regions are unexcitable, and that the current merely passes through them. It is, a priori, more likely that there is also functional differentiation of the cortical centres to which they are distributed, and that the grey matter is, under normal conditions, the natural excitant of the reactions which we are able to produce by artificial stimulation with the electric current. And a comparison of the respective reactions of the cortex and medullary fibres indicates such differences as can only be explained on the supposition that the cortical centres are themselves excitable.

First, as regards the relative excitability of grey matter and subjacent medullary fibres. This is a point on which there are some differences of opinion, but Putnam found ${ }^{37}$ the medullary fibres less excitable than the cortex, so that in order to produce the customary reaction it was necessary to use a much stronger current than before. This has been confirmed by Franck and Pitres, who have further shown that the diminished excitability cannot be accounted for by mere shock or hæmorrhage, inasmuch as the neighbouring grey matter acted as readily as before. They have further given reasons for believing that the contrary results obtained by Richet, ${ }^{38}$ and Bubnoff and Heidenhain ${ }^{39}$ are due to the action on the cortex of the chloral and morphine under which their experiments were performed. These agents, without doubt, paralyse the excitability of the grey matter. It was noted by Fritsch and Hitzig in their experiments that the anodal closure was a more effective stimulus than the cathodal, a fact which might be interpreted as signifying that the real stimulus proceeded from the virtual cathode in the deep layers of the cortex, or termination of the medullary fibres. This, however, has been shown by

33 Examen de quelques Points de la Physiologie du Cerveau, 1873 31 Proceedings of the Royal society, June, 1874. 3is Comptes Rendus Société de Biologie, 1877 . 36 Proceedings of the Royal Society, No. 286,1890 37 Boston Med. and Surg. Journal, 1874 . 8 sur les Circonvolutions Cérébrales, 1879

'39 Pfiuger's Archiv f. Physiologie, 1881.
Gerber ${ }^{4}$ not to be uniformly the case. Gerber finds that when the cortex is in a normal state the cathode is the more effective stimulant, but that, when changes have occurred from long exposure, the anode predominates. These experiments would, therefore, indicate that in the normal condition of the brain the laws of galvanic excitability are the same as for motor nerves.

Another important difference between the reactions of the cortex and the subjacent medullary fibres, which was first pointed out by Franck and Pitres, is that the time lost between the application of the stimulus and the occurrence of muscular contraction is much greater in the case of the former than the latter. This interval, after deducting the time necessary for the transmission of the impulse through the spinal cord and motor nerves, indicates a retardation in the cortex of 0.045 second. After removal of the grey matter and application of the electrodes to the medullary fibres, the period of retardation diminishes to 0.030 second-that is, about one-third less, - and this difference is put at a considerably higher figure by Bubnoff and Heidenhain. The signification of this fact is that the grey matter of the cortex does not behave like an inert layer, which merely allows transmission of the electric current to the medullary fibres, but, like other nerve centres, stores up and transforms the stimuli which it has received into its own energy.

There is also a characteristic difference between the muscular curves registered on stimulation of the cortex and medullary fibres respectively. In the latter case the curve rises abruptly, and is of short duration; while in the former it rises more gradually, is more prolonged, and frequently marked by the occurrence of a secondary tetanus, which latter is altogether peculiar to the cortex, and is never seen when the medullary fibres alone are stimulated. The cortex is apt, after repeated stimulation, or after the receipt of a succession of stimuli, each insufficient to produce reaction, to respond by tonic, followed by clonic, spasms of the correlated muscles of a truly epileptic type. These convulsions tend to spread and become generalised in the order and sequence originally described by Hughlings Jackson. They never vccur on stimulation of the medullary fibres alone, apart from the intervention of the grey matter of the cortex on the one side or the other, and cannot be produced if the cortical centres are entirely destroyed on both sides. The duration of the effects of stimulation of the medullary fibres is strictly proportional to that of the stimulus which is applied to them. We shall also see as we proceed that the effects of localised destruction of the cortex are the counterpart of those of irritation, however induced, and we mav from this conclude that there is the same functional differentiation in the cortex as in the medullary fibres, even if the facts which have just mentioned should not be regarded as of themselves completely establishing this proposition.

\section{TWO CASES OF INTUSSUSCEPTION TREATED WITH THE AID OF BARNES' BAG;}

WITH REMARKS.

BY WALTER RIVINGTON, B.A., M.B. \& M.S.LOND.; F.R.C.S. ENG.,

CONSULTING SURGEON TO THE LONDON HOSPITAL.

OF the two cases which I propose to relate, for the purpose of showing that Barnes' bag may sometimes be usefully employed for reducing and preventing the return of an intussusception, the first occurred in 1877. The patient, a man of fifty-seven, was in the London Hospital under my colleague, Dr. Stephen Mackenzie. Six months before admission he had noticed that there seemed to be a constriction in his rectum. $\mathrm{He}$ passed his fæces in small lumps, streaked with blood. The bleeding increased, until it amounted, according to his estimate (which was probably excessive) to as much as a teacupful three or four times daily. These symptoms subsided under treat-

40 Beitrïge zur Lehre von der electrischen Reizuno des Grosshirns Pfluger's Archiv fuir Physiologie, Band xxxix., 1888.

$$
\text { ¿ } 2
$$


ment, but were followed by loose motions and by two attacks of bleeding and pain. During the later attack he felt the gut protrude externally for two inches, and then return. 'This happened three times in half an hour, and the pain was excruciating. The pain and bleeding continued up to admission, seven days from the outset of the attack, and a slimy discharge took place from the rectum. On admission the patient was a pale, cachectic, wasted man. The ubdomen was distended, tympanitic, and tender, especially in the hypogastric region. No tumour could be felt in the abdomen. On rectal exanination a rounded, firm swelling about the size of a hen's egg, with a velvety surface, was detected. The finger could be passed ail round it, and at its apex was an orifice into which the finger could be readily passed. Examination with the speculum showed that the mucous membrane was deeply congested. There could be no doubt that an intussusception existed, and Dr. Mackenzie, whose description I have followed, asked me to see the case and treat it. As the intussusception was within reach, and, from the absence of abdominal tumour, appeared likely to be of limited extent, I thought that it would very probably yield to the equable pressure which could be exercised on it by distending a Barnes' bag with fluid after introducing it into the rectum empty, and so it proved. The intussusception gradually receded, and finally disappeared altogether. "With reposition of the bowel the patient passed a quantity of flatus and liquid freces, and obtained great relief. A swelling was noticed in the left inguinal region, but this disappeared in a few days. He remained in the hospital a couple of months; the hæmorrhage and slimy discharge disappeared, but his motions were nearly always liquid or semi-solid, and he suffered from flatulence and crampy pains." He continued under Dr. Mackenzie's observation until his death, about a year and a half later. The diagnosis which Dr. Mackenzie formed at the time when the patient was in the hospital was "annular stricture of the descending colon, leading to prolapse of the bowel through the straining efforts necessary to overcome the obstruction" "1 and at the post-mortem which Dr. Mackenzie obtained at the Leytonstone Workhouse he found primary cancer of the sigmoid flexure and secondary cancer of the peritoneum and liver.

The second case occurred recently. Alfred B-, seven months old, was brought to the receiving-room at the London Hospital by his mother on Sunday, Nov. 17th, 1889. She said that the bowel had come down, and on examination by my house surgeon, Mr. Hicks, the child was found to be suffering from intussusception, and was at once admitted into the wards. The mother stated that about a fortnight ago she had noticed one morning that the child seemed to be in great pain, very restless, crying incessantly, and vomiting. About four o'clock in the afternoon of that day the child passed a quantity of blood. The mother at once took him to a local doctor, who treated him up to his admission into the hospital. Blood continued to flow from the rectum for three days, and then stopped, exces. sive diarrhoea taking its place. Diarrhoea and vomiting continued till the child was brought to the hospital. On the previous day the bowel had descended, and the mother had pushed it back; but as it would not remain up she thought it best to apply to the hospital for relief. After admission Mr. Hicks saw the child, and reduced the intuswusception by injecting two pints of water with a Higginson's syringe. The injection was effectual for a time, but the next morning the bowel was down again. It was noticed that the ileo-cæeal valve formed the apex of the intussuscepted portion of bowel which projected considerably beyond the anus. Reduction of the intussusception was easy as far as the upper part of the rectum ; but all attempts to reduce it further by nteans of injections of fluid and insufflation aided by position failed, as shown, on examination of the abdomen, by the persistence of a characteristic tumour in the position of the descending colon and sigmoid flexure. As often as the bowel descended it was replaced and retained by strapping the buttocks together. In spite, however, of all that could be done, it constantly recurred, and on Nov. 22nd I made an ineffectual attempt to reduce it by injections and insutflation, aided by suspension of the child by his legs. Finding that I could not succeed in this way, I reduced the intussusception as far as it would go, and retained it by the introduction into the rectum of an empty Barnes' bag, which was then distended with air. Instruc tions were given that the bag should be removed twice a day to allow the escape of fiquid motions. My primary object was to prevent the bowel from descending, with a view to favouring further efforts at reduction, and 1 hoped that the peristaltic action of the intestine, exerted fruitless] for the expulsion of the bag, might act in the opposite direction, and assist the devolution of the involved intestine. During the first day little impression was made, for the bowel descended when the bas was removed, and I then harl the bag replaced and retained for twenty four hours. At the end of that time the tumour had disappeared, and on taking away the bag a large quantity of flatus escaped. On Dec. 4th the notes state that "the patient has from day to day been gradually getting better from the intussusception, passing fairly good motions." On only one occasion subsequently to the reduction was any blood passed; and as the abdomen was flaccid, and no tumour could be felt on the most careful examination, this escape was attributed either to some remaining congestion of the previously engaged bowel or to ulceration. The child continued regularly to pass motions, was free from sickness, and took the breast as usual, but he began to emaciate. A large abscess formed in the neck and was opened by Mr. Gedre. An extensive purpuric patch appeared on the left side of the abdomen, reaching from the ribs to the crest of the ilium, with small spots and petechiæe around it, and another large patch occupied the left side of the neck. The abdomen was retracted; there was no trace of any tumour on either side; the protrusion of gut did not return, and the bowels were regularly relieved. Nevertheless the child became thinner and weaker, and died on Dec. 13th, twenty-six days after admission, and nearly three weeks after the reduction of the intussusception.

The mother refused to allow any inspection, even of the abdomen only, notwithstanding all persuasion, and thus the conclusive demonstration of the efficacy of the treatment and the explanation of the cause of death are necessarily wanting. I cannot doubt, however, that the intussuscep tion was reduced, because no tumour could be detected, natural motions free from blood were passed, there was no sickness, and the child was free from pain. Granting this, the question arises what was the modus operand of the distended bag in the reduction of the intussusception? Two explanations present themselves. The first is that the reduction was due to the peristaltic or antiperistaltic action of the bowel which contained the intus susception, and this appears to me to be exceedingly probable. A second idea which occurred to us was that the reduction might be due to the accumulation of gas above the bag. The bowel not being sufficiently nipped to prevent flatus from passing, it would escape through the apex of the intussusception, and then collect between the intussus cipient and intussuscepted portion, and so exert the same intluence as artificial insufllation. If this view be tenable the method of reduction may be described as the method of natural insufflation through the agency of Barnes' bag. Whichever may be the correct explanation, it is apparent that the use of the bag was very different in the second case from its $u s e$ in the first. The bag in the first case was simply a convenient method of applying pressure. It acted in the same way as injections or insufflation, but more expeditionsly, with less risk to the bowel, and with greater accuracy and precision. In the second case it acted indirectly by blocking the canal, forming a point d'appui for the peristaltic contraction of the muscular fibres of the intestine, and preventing the exit of flatus. It also prevented the protrusion of the bowel from the rectum, and, if it had done nothing else, would have enabled us to renew our efforts at reduction by injections, insufflation, manipulation, and position, with improved prospects of success. The cases in which this adjunct to our methods of treating intussusception may be found serviceable are clearly not the cases of acute strangulation, in which nothing can pass through the engaged intestine, or cases where adhesions have formed, but those which are also open to treatment by injections and insufflation, but which readily recur on replacement or do not disappear entirely under injections of air or fluid. In these subacute or chronic cases a trial of the method would not prejudice any other measures to which, in the result of failure, it might seem advisable to resort. It may also be useful in keeping up trou blesome prolapse of the mucous membrane of the rectum. Finsbury-square, E.C. 